\title{
A Jacobian elliptic single-field inflation
}

\author{
J. R. Villanueva ${ }^{1,2, a}$, Emanuel Gallo $^{3,4, b}$ \\ ${ }^{1}$ Instituto de Física y Astronomía, Universidad de Valparaíso, Gran Bretaña 1111, Valparaiso, Chile \\ ${ }^{2}$ Centro de Astrofísica de Valparaíso, Gran Bretaña 1111, Playa Ancha, Valparaiso, Chile \\ ${ }^{3}$ FaMAF, Universidad Nacional de Córdoba, Ciudad Universitaria, 5000 Córdoba, Argentina \\ ${ }^{4}$ Instituto de Física Enrique Gaviola (IFEG), CONICET, Ciudad Universitaria, 5000 Córdoba, Argentina
}

Received: 30 March 2015 / Accepted: 18 May 2015 / Published online: 10 June 2015

(C) The Author(s) 2015. This article is published with open access at Springerlink.com

\begin{abstract}
In the scenario of single-field inflation, this field is described in terms of Jacobian elliptic functions. This approach provides, when constrained to particular cases, analytic solutions already known in the past, generalizing them to a bigger family of analytical solutions. The emergent cosmology is analyzed using the Hamilton-Jacobi approach and then the main results are contrasted with the recent measurements obtained from the Planck 2015 data.
\end{abstract}

\section{Introduction}

One of the main paradigms of the early universe is to the socalled inflation, which, for example, allows quantum fluctuations to produce the seeds for the cosmic microwave background anisotropy and large scale structure, so one of the ways by which this stage can emerge is, for example, protecting the holographic principle [1]. In the standard inflationary cosmology, the universe is dominated by a scalar inflaton field, $\phi$, self-interacting through a potential, $V(\phi)$, with an energy density $\rho_{\phi}=\frac{1}{2} \dot{\phi}^{2}+V(\phi)$, and pressure $p_{\phi}=\frac{1}{2} \dot{\phi}^{2}-V(\phi)$. Since inflation proceeds if the potential energy of the field dominates its kinetic energy, the weak energy condition ensures that the pressure is negative during the inflationary process allowing for an extremely rapid expansion of the universe. In general, it is not easy to obtain exact solutions of the Einstein equations with an inflaton field as source, for a given potential $V(\phi)$. However, for some simple cases, exact solutions can be obtained without even making use of the slow roll approximation.

To perform a quantitative analysis of the inflation one could proceed in two way: using the standard slow-roll approximation [2], or using the Hamilton-Jacobi method [36]. In this work, we opt for the latter approach, obtaining new exact solutions for an inflaton field where the potential is

\footnotetext{
a e-mail: jose.villanuevalob@uv.cl

b e-mail: egallo@famaf.unc.edu.ar
}

expressed in terms of elliptical functions. The reason behind this choice is the fact that many authors have reported potentials in terms of trigonometric [7-9] and hyperbolic functions [7-14]. Within this context, and keeping in mind that both, trigonometric and hyperbolic functions, are nothing but particular cases of elliptical functions, we seek for a generalization based on functions of this kind.

This paper is organized as follows: in Sect. 2 we give a review of the Hamilton-Jacobi approach to inflation. In Sect. 3 we present a quartic potential which can be expressed in terms of elliptical functions, and we provide the general solutions and the expresions for the relevant cosmological parameters. In Sect. 4 we study scalar and tensor perturbations and we also compare them with the available observational data.

\section{Hamilton-Jacobi approach to inflation}

The starting point is the expression of the equations governing the background in terms of the inflaton $\phi$, so in units where $\hbar=c=1$ these relations are given by

$H^{2}=\frac{8 \pi}{3 m_{p}^{2}}\left[\frac{1}{2} \dot{\phi}^{2}+V(\phi)\right]$

and

$\ddot{\phi}+3 H \dot{\phi}+V^{\prime}(\phi)=0$,

where $m_{p}=G^{-1 / 2}$ is the Planck mass, a dot represents differentiation with respect to the cosmic time, $t$, and a prime represents differentiation with respect to the inflaton scalar field. Taking the temporal derivative of Eq. (1), we obtain

$H \dot{H}=\frac{4 \pi}{3 m_{p}^{2}}\left[\ddot{\phi}+V^{\prime}(\phi)\right] \dot{\phi}$, 
so, using Eq. (2) into Eq. (3) leads to the useful relation

$\dot{\phi}^{2}=-\left(\frac{m_{p}^{2}}{4 \pi}\right) \dot{H}$.

Also, considering the relation between operators

$$
\frac{\mathrm{d}}{\mathrm{d} t}=\dot{\phi} \frac{\mathrm{d}}{\mathrm{d} \phi}
$$

Equation (4) can be written as

$$
\dot{\phi}=-\left(\frac{m_{p}^{2}}{4 \pi}\right) H^{\prime}
$$

If we assume that the Hubble parameter and the inflaton are invertible, then we can write the potential as a function of $H$. Therefore, inserting Eq. (6) into Eq. (1) we obtain the kinematic equation involving the evolution of the scalar field

$$
\left(\frac{\mathrm{d} H}{\mathrm{~d} \phi}\right)^{2}=\frac{12 \pi}{m_{p}^{2}} H^{2}-\frac{32 \pi^{2}}{m_{p}^{4}} V .
$$

Since the inflaton is a real scalar field, the condition that $H^{2}>\frac{8 \pi}{3 m_{p}^{2}} V$ must be fulfilled during inflation. Equations (6) and (7) are called the Hamilton-Jacobi equations, and they dictate the kinematic evolution. In order to obtain exact solutions to Eqs. (6) and (7), we propose a potential that leads to analytic solutions, i.e., that allows us to find the generating function, $H(\phi)$. Thus, the set of Eqs. (6) and (7) yield exact solutions. On the other hand, from Eq. (6), $a^{\prime} H^{\prime}=-\left(\frac{m_{p}^{2}}{4 \pi}\right) a H$, it follows that

$a(\phi)=a_{i} \exp \left[-\frac{4 \pi}{m_{p}^{2}} \int_{\phi_{i}}^{\phi} \frac{H(\phi)}{H^{\prime}(\phi)} \mathrm{d} \phi\right]$,

where $a_{i}=a\left(\phi_{i}\right)$, and, from now on, the subscripts $i$ and $f$ imply that quantities are evaluated when inflation begins and ends, respectively. The above equation implies that the scalar factor $a$ can be written as a function of the inflaton field $\phi$, and thus, assuming that we know the scalar field as a function of time, we can obtain the scale factor as a function of the cosmological time.

It is also possible to express the acceleration equation for the scale factor as

$\frac{\ddot{a}}{a}=H^{2}\left(1-\epsilon_{H}\right)$,

where the first Hubble hierarchy parameter $\epsilon_{H}$ is given by

$\epsilon_{H} \equiv-\frac{\mathrm{d} \ln H}{\mathrm{~d} \ln a}=\left(\frac{m_{p}^{2}}{4 \pi}\right)\left(\frac{H^{\prime}}{H}\right)^{2}$.
This parameter provides information as regards the acceleration of the universe, so during inflation the bound $\epsilon_{H} \ll 1$ is fulfilled, and inflation ends when $\epsilon_{H}=1$.

Another quantity to describe inflation is the number of e-foldings of the physical expansion, which is given by

$N \equiv \ln \left(\frac{a_{f}}{a_{i}}\right)$,

so, using Eqs. (6), (8), and (10) we obtain

$N \equiv \int_{t_{i}}^{t_{f}} H \mathrm{~d} t=\left(\frac{4 \pi}{m_{p}^{2}}\right) \int_{\phi_{f}}^{\phi_{i}} \frac{H}{H^{\prime}} \mathrm{d} \phi=\int_{\phi_{f}}^{\phi_{i}} \frac{1}{\epsilon_{H}} \frac{H^{\prime}}{H} \mathrm{~d} \phi$.

In the description of inflation it is also relevant to show that the solutions are independent from their initial conditions. This guarantees the true predictive power that any inflationary universe model must have, otherwise the corresponding physical quantities associated with the inflationary phase, such as the scalar or tensor spectra, would depend on these initial conditions. Thus, any inflationary model needs to fulfill the condition that its solutions follow an attractor behavior, in the sense that solutions with different initial conditions should converge to a unique solution [3].

Let us start by considering a linear perturbation, $\delta H(\phi)$, around a given inflationary solution, expressed by $H_{(0)}(\phi)$. In the following we will refer to this quantity as the background solution, and any quantity with the subscript (0) is assumed to be evaluated taking into account the background solution. Substituting $H=H_{(0)}+\delta H$ into Eq. (7) and then linearizing, it is straightforward to show that $[2,15,16]$

$\delta H \simeq \frac{m_{p}^{2}}{12 \pi} \frac{H_{(0)}^{\prime}}{H_{(0)}} \delta H^{\prime}$

which can be solved as

$\delta H(\phi)=\delta H\left(\phi_{i}\right) \exp \left[\int_{\phi_{i}}^{\phi}\left(\frac{3}{\epsilon_{H}}\right) \frac{H_{(0)}^{\prime}}{H_{(0)}} \mathrm{d} \phi\right]$,

where $\phi_{i}$ corresponds to some arbitrary initial value of $\phi$. Since $\mathrm{d} \phi$ and $H^{\prime}$ have opposite signs (assuming that $\dot{\phi}$ does not change sign due to the perturbation $\delta H$ ) the linear perturbations tend to vanish quickly [2].

\section{A quartic potential}

In this section we will present a class of potential which can be given in terms of elliptic functions. The reason behind this choice is the fact that many authors have reported potentials in terms of trigonometric [7-9] and hyperbolic functions 
[7-14]. So, recalling that both functions, trigonometric and hyperbolic, are special cases of elliptic functions, we will try to find a potential which leads to some function of this kind. To proceed, we define the following potential:

$\frac{8 \pi}{3 m_{p}^{2}} V=\alpha_{0}+\alpha_{1} Y(H)+\alpha_{2} Y^{2}(H)+\alpha_{4} Y^{4}(H)$,

where the $\alpha_{n}$, for $n=1,2,3,4$, are explicitly given by $\alpha_{0}=$ $H_{0}^{2}-H_{1}^{2}\left(1-k^{2}\right), \alpha_{1}=2 H_{0} H_{1}, \alpha_{2}=2 H_{1}^{2}\left(1-k^{2}\right), \alpha_{4}=$ $H_{1}^{2} k^{2}$, and the dimensionless function $Y$ is given by

$Y(H)=\frac{H}{H_{1}}-\mathcal{H}$.

In these definitions, $\mathcal{H} \equiv H_{0} / H_{1}, H_{1}$, and $k$ are constants which will be tested against observational data.

Substitution of Eq. (15) into the general Eq. (7) implies that

$$
\left(\frac{1}{\beta} \frac{\mathrm{d} Y}{\mathrm{~d} \phi}\right)^{2}=\left(1-Y^{2}\right)\left(k^{\prime 2}+k^{2} Y^{2}\right),
$$

where $\beta^{2}=12 \pi / m_{p}^{2}$ and $k^{\prime 2}=1-k^{2}$. Therefore, performing an integration (see [17-21]), and then solving for $H$, we obtain

$H(\phi)=H_{1}\left\{\mathcal{H}+\operatorname{cn}\left[\beta\left(\phi-\phi_{0}\right)\right]\right\}$,

where $\operatorname{cn}(x) \equiv \operatorname{cn}(x \mid k)$ is the Jacobi elliptic cosine function. Figure 1 depicts $H(\phi)$ for different values of the modulus $k$. So, by defining the dimensionless scalar field $\Phi=$ $\beta\left(\phi-\phi_{0}\right)$, and after a slight manipulation, the potential is conveniently written as

$\mathcal{V}(\phi) \equiv \frac{8 \pi}{3 m_{p}^{2}} \frac{V}{H_{1}}=(\mathcal{H}+\mathrm{cn} \Phi)^{2}-\operatorname{sn}^{2} \Phi \mathrm{dn}^{2} \Phi$,

where $\operatorname{sn}(x) \equiv \operatorname{sn}(x \mid k)$ is the Jacobi elliptic sine function, and $\operatorname{dn}(x) \equiv \operatorname{dn}(x \mid k)$ is the Jacobi elliptic delta function. The behavior of $V(\phi)$ is indicated in Fig. 2, where for comparison, different values of the modulus $k$ are shown. The dimensionless pressure and the energy density are given by

$\bar{\rho}_{\phi} \equiv \frac{8 \pi}{3 m_{p}^{2}} \frac{\rho_{\phi}}{H_{1}^{2}}=(\mathcal{H}+\mathrm{cn} \Phi)^{2}$

and

$\bar{p}_{\phi} \equiv \frac{8 \pi}{3 m_{p}^{2}} \frac{p_{\phi}}{H_{1}^{2}}=-(\mathcal{H}+\mathrm{cn} \Phi)^{2}+\operatorname{sn}^{2} \Phi \mathrm{dn}^{2} \Phi$,

respectively. Therefore, the parameter of the equation of state, $\omega_{\phi}\left(\rho_{\phi}\right)=p_{\phi} / \rho_{\phi}$, can be written as

$\omega_{\phi}\left(\rho_{\phi}\right)=-1+\sqrt{f_{\phi}}$,

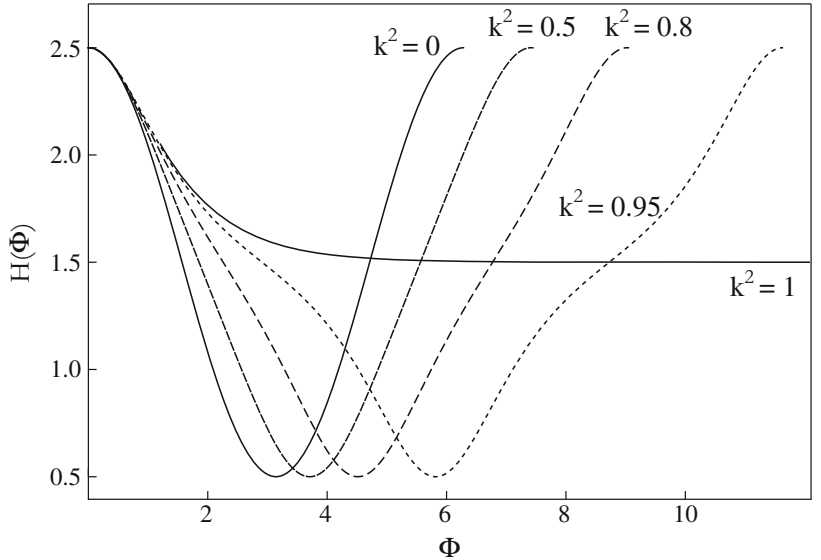

Fig. 1 Plot of the Hubble parameter $H$ as a function of the dimensionless scalar field $\Phi$

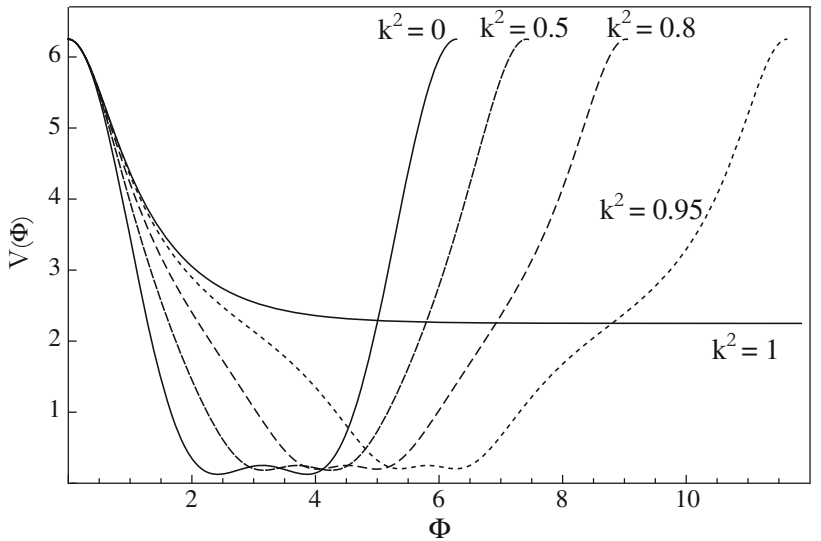

Fig. 2 The characteristic behavior of the potential $V$ as a function of the dimensionless scalar field $\Phi$ for different values of the modulus $k$. All plots were made using $\mathcal{H}=1.5$

where the function $f_{\phi} \equiv f\left(\rho_{\phi}\right)$ is given explicitly by

$\frac{\left(1-\mathcal{H}^{2}+2 \mathcal{H} \rho^{1 / 2}-\rho\right)\left[1-k^{2}\left(1-\mathcal{H}^{2}+2 \mathcal{H} \rho^{1 / 2}-\rho\right)\right]}{\rho^{2}}$.

Note that the condition $f_{\phi} \geq 0$ must be satisfied for every pair $(\mathcal{H}, k)$.

Also, from Eq. (22) we can see that the Jacobian scalar field $\phi$ is driving inflation in such way that the condition $\omega_{\phi} \leq$ -1 always is satisfied. Clearly there are four critical values for the (dimensionless) density which lead to a cosmologicallike constant regime $\left(\omega_{\phi}=-1\right)$ :

$\bar{\rho}_{\phi, 1,2}^{(c)}=(\mathcal{H} \pm 1)^{2}$,

$\bar{\rho}_{\phi, 3,4}^{(c)}=\mathcal{H}^{2}+\left(1-\frac{1}{k^{2}}\right) \pm 2 \sqrt{\mathcal{H}^{2}\left(1-\frac{1}{k^{2}}\right)}$.

First of all, $\bar{\rho}_{\phi, 1}^{(c)}$ and $\bar{\rho}_{\phi, 2}^{(c)}$ are positive and independent of the modulus $k$. But, since $1 \geq k \geq 0, \bar{\rho}_{\phi, 3}^{(c)}$ and $\bar{\rho}_{\phi, 4}^{(c)}$ are complex 


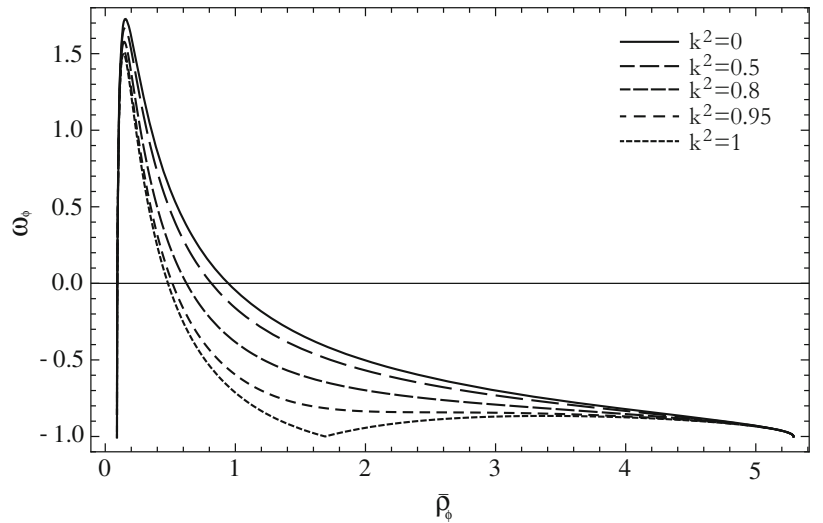

Fig. 3 Plot of the parameter of the equation of state $\omega_{\phi}$ as a function of the dimensionless density $\bar{\rho}_{\phi}$ for different values of the modulus $k$. All plots were made using $\mathcal{H}=1.3$

(a conjugate pair) except in the case $k=1$, where they take the value (degenerate) $\bar{\rho}_{\phi, 1}^{(c)}=\bar{\rho}_{\phi, 2}^{(c)}=\mathcal{H}^{2}$; see Fig. 3 .

Using the generating function (18) in Eq. (8), and then performing an integration, we find

$\frac{a(\phi)}{a_{i}}=\frac{\operatorname{sd} \Phi}{\operatorname{sd} \Phi_{i}}\left(\frac{\mathcal{G}(\phi)}{\mathcal{G}\left(\phi_{i}\right)}\right)^{\mathcal{H}}$

where $\operatorname{sd}(x \mid k)=\operatorname{sn}(x \mid k) / \operatorname{dn}(x \mid k)$, and the Gugu function $\mathcal{G}(\phi)$ is given by

$\mathcal{G}(\phi)=\frac{1+\operatorname{cn} \Phi}{\operatorname{sn} \Phi} e^{\frac{k}{k^{\prime}} \arctan \left(\frac{k}{k^{\prime}} \operatorname{cn} \Phi\right)}$.

Since this function diverges when $k=1$ (or $k^{\prime}=0$ ) we must exclude it and integrate directly this case from Eq. (8), obtaining

$\frac{a(\phi)}{a_{i}}=\frac{\sinh \Phi}{\sinh \Phi_{i}}\left(\frac{\mathcal{F}(\phi)}{\mathcal{F}\left(\phi_{i}\right)}\right)^{\frac{H_{0}}{H_{1}}}$,

with the function $\mathcal{F}(\phi)$ defined by

$\mathcal{F}(\phi)=\frac{\cosh \Phi-1}{\sinh \Phi} e^{\cosh \Phi}$.

In the same way, using the fact that $H^{\prime}(\phi)=-\beta H_{1} \operatorname{sn} \Phi$ dn $\Phi$ together with Eq. (6), we see that the cosmological time is $t=\ln \mathcal{G}^{\frac{1}{H_{1}}}(\phi)$, or more conveniently

$\mathcal{G}(\phi)=e^{H_{1} t}$,

if $0 \leq k<1$, whereas in the hyperbolic limit, $k=1$, the cosmological time is $t=\ln \mathcal{F}^{\frac{1}{H_{1}}}(\phi)$, or

$\mathcal{F}(\phi)=e^{H_{1} t}$.

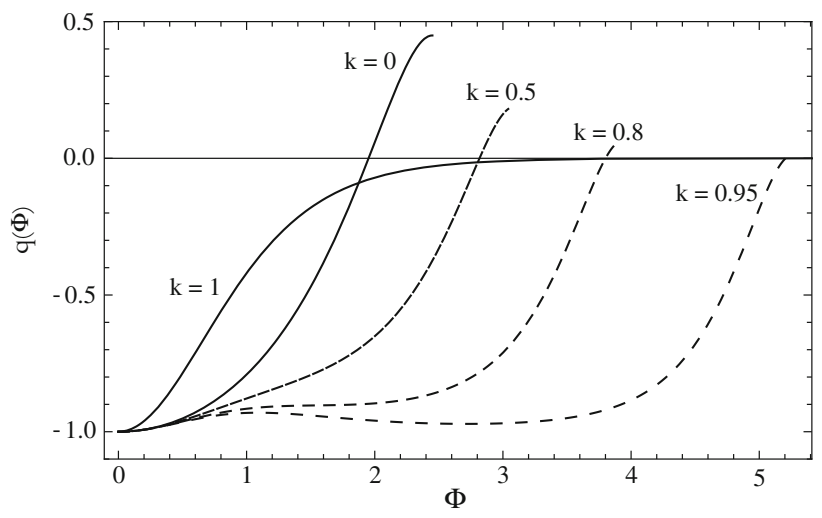

Fig. 4 Plot of the deceleration parameter $q$ as a function of the dimensionless scalar field $\Phi$

Note from Eqs. (26), (30), and (31) we note that $a \propto$ $e^{H_{0}\left(t-t_{i}\right)}$, required by the de Sitter stage.

In addition, making use of the properties of the Jacobian elliptic functions, we can write the first Hubble hierarchy parameter (10) in the convenient form

$\epsilon_{H}=\frac{\operatorname{sn}^{2} \Phi \mathrm{dn}^{2} \Phi}{[\mathcal{H}+\mathrm{cn} \Phi]^{2}}$.

In the limit $k \rightarrow 0$, this simplifies to

$\epsilon_{H}=\frac{\sin ^{2} \Phi}{[\mathcal{H}+\cos \Phi]^{2}}$

while in the limit $k \rightarrow 1$, we obtain

$\epsilon_{H}=\frac{\operatorname{sech}^{2} \Phi \tanh ^{2} \Phi}{[\mathcal{H}+\operatorname{sech} \Phi]^{2}}$

Let us stop briefly in this last expression for the case $\mathcal{H}=$ $H_{0}=0$, which, except for a constant, leads to the expression obtained by del Campo [12] when a Chaplygin-like scalar field is considered.

For the case of inflation $(\ddot{a} / a>0)$, the relevant boundary condition is de Sitter expansion, $\epsilon_{H}\left(\phi_{S}\right)=0$. So, for our case this condition leads to $\phi_{S}=\phi_{0}$. From the definition of $\epsilon_{H}$, this is equivalent to $H^{\prime}(\phi)=0$, and it follows that $\phi_{0}$ is a stationary point of the field:

$\dot{\phi}_{l_{\phi=\phi_{0}}}=-\frac{m_{p}^{2}}{4 \pi} H^{\prime}\left(\phi_{0}\right)=0$.

From the generating function (18) we see that this point is $\phi_{0}=0$. Note that at this point the Hubble parameter is given by $H\left(\phi_{0}\right)=H_{0}+H_{1}$.

In order to assess whether we have an inflationary period, we introduce the deceleration parameter $q$, defined as $q=$ $-\frac{\ddot{a} a}{\dot{a}^{2}}$, which, in terms of the first Hubble hierarchy parameter $\epsilon_{H}$, becomes $q=\epsilon_{H}-1$, which gives 
Table 1 Values for the dimensionless scalar field for some values of the modulus $k$. The values of $\Phi_{f}$ were calculated using the condition $\epsilon_{H}\left(\Phi_{f}\right)=1$ from Eq. (32), while the values of $\Phi_{i}$ were calculated using the condition $N\left(\Phi_{i}\right)=60$ and $N\left(\Phi_{i}\right)=70$ from Eq. (37). In all these computations we use $\mathcal{H}=1.3$, except for $k=1$ where we have assumed $\mathcal{H}=0$

\begin{tabular}{|c|c|c|c|c|c|}
\hline \multirow[t]{2}{*}{$k^{2}$} & \multirow[t]{2}{*}{$\Phi_{f}$} & \multicolumn{2}{|l|}{$N=60$} & \multicolumn{2}{|l|}{$N=70$} \\
\hline & & $\Phi_{i}$ & $\Delta \Phi$ & $\Phi_{i}$ & $\Delta \Phi$ \\
\hline 0 & 1.9515 & 1.5764 & 0.3752 & 1.5757 & 0.3760 \\
\hline 0.5 & 2.8170 & 1.6883 & 1.1287 & 1.6863 & 1.1307 \\
\hline 0.8 & 4.0568 & 2.0118 & 2.045 & 2.0088 & 2.0480 \\
\hline 0.95 & 5.3123 & 2.6781 & 2.6342 & 2.6747 & 2.6376 \\
\hline 1 & 19.5933 & 1.1206 & 18.4728 & 1.0845 & 18.5088 \\
\hline
\end{tabular}

$q=-1+\frac{\mathrm{sn}^{2} \Phi \mathrm{dn}^{2} \Phi}{[\mathcal{H}+\mathrm{cn} \Phi]^{2}}$

The behavior of $q(\phi)$ is indicated in Fig. 4 for different values of the modulus $k$.

The number of the e-folds is found to be

$N\left(\Phi_{i}\right)=\ln \left(\frac{\operatorname{sd} \Phi_{f}}{\operatorname{sd} \Phi_{i}}\right)+\mathcal{H} \ln \left(\frac{\mathcal{G}\left(\Phi_{f}\right)}{\mathcal{G}\left(\Phi_{i}\right)}\right)$

We must remark that the final value of the dimensionless scalar field $\Phi_{f}$ is obtained using the transcendental equation

$\mathcal{H}=-\operatorname{cn} \Phi_{f} \pm \operatorname{sn} \Phi_{f} \operatorname{dn} \Phi_{f}$

From Eqs. (37) and (38) using $N=60, N=70$, $\mathcal{H}=1.3$ (except for $k=1$ where we have used $\mathcal{H}=0$ ) and different values of the modulus $k$, some values of $\left(\phi_{i}, \phi_{f}\right)$ are presented in Table 1.

The study of the attractor behavior of the model is performed by considering a linear perturbation, $\delta H(\phi)$, around a given solution and following the steps described above. Thus, from Eqs. (14), (18), and (32), we get

$\delta H(\phi)=\left[\frac{\operatorname{sd} \Phi_{i}}{\operatorname{sd} \Phi}\left(\frac{\mathcal{G}\left(\phi_{i}\right)}{\mathcal{G}(\phi)}\right)^{\mathcal{H}}\right]^{3} \delta H\left(\phi_{i}\right)$.

Since the factor accompanying $\delta H\left(\phi_{i}\right)$ is nothing but $\left(a / a_{i}\right)^{-3}$ [see the expression (26)], which during inflation increases at least 70 e-fold, we can conclude that $\delta H(\phi)$ is very small.

\section{Scalar and tensor perturbations}

In the inflationary scenario, the quantum fluctuations are found to be relevant because they generate two important types of perturbations: density perturbations (arising from quantum fluctuations in the scalar field, together with the corresponding scalar metric perturbations [22-24]), and relic gravitational waves (which are tensor metric fluctuations [25-29]). The former is sensitive to gravitational instability and leads to structure formation [30], while the latter predicts a stochastic background of relic gravitational waves which could influence the cosmic microwave background anisotropy via the presence of polarization [31,32].

In order to describe these perturbations we introduce a series of parameters known as the Hubble hierarchy parameters. We have already defined one of them through Eq. (10), presented as the first Hubble hierarchy parameter. The second Hubble hierarchy parameter, $\eta_{H}$, is defined by

$\eta_{H} \equiv-\frac{\mathrm{d} \ln H^{\prime}}{\mathrm{d} \ln a}=\left(\frac{m_{p}^{2}}{4 \pi}\right)\left(\frac{H^{\prime \prime}}{H}\right)$,

where plugging in the Jacobian scalar field leads to

$\eta_{H}=\frac{\operatorname{cn} \Phi\left(1-2 \mathrm{dn}^{2} \Phi\right)}{\mathcal{H}+\operatorname{cn} \Phi}$.

The third Hubble hierarchy parameter, $\xi_{H}^{2}$ is defined by

$\xi_{H}^{2} \equiv\left(\frac{m_{p}^{2}}{4 \pi}\right)^{2}\left(\frac{H^{\prime \prime \prime} H^{\prime}}{H^{2}}\right)$,

leading to

$\xi_{H}^{2}=\frac{\operatorname{sn}^{2} \Phi \mathrm{dn}^{2} \Phi\left(6 k^{2} \mathrm{sn}^{2} \Phi-4 k^{2}-1\right)}{[\mathcal{H}+\mathrm{cn} \Phi]^{2}}$.

The quantum fluctuations produce a power spectrum of scalar density fluctuations of the form $[33,34]$

$\mathcal{P}_{\mathcal{R}}(\widehat{k})=\left.\left(\frac{H}{|\dot{\phi}|}\right)^{2}\left(\frac{H}{2 \pi}\right)^{2}\right|_{a H=\widehat{k}}$.

This perturbation is evaluated when a given mode $\widehat{k}$ crosses the horizon during inflation, i.e. at $a H=\widehat{k}$. These modes do not evolve outside the horizon, so we can assume they keep a fixed value after crossing the horizon during inflation. In order to obtain some comparison with the available observational data, we introduce the scalar spectral index $n_{s}$ defined as

$n_{s}-1 \equiv \frac{\mathrm{d} \ln \mathcal{P}_{\mathcal{R}}}{\mathrm{d} \ln \widehat{k}}$

After a brief calculation, one obtains

$n_{s}=1-4 \epsilon_{H}+2 \eta_{H}$,

so, using Eqs. (32) and (41), the spectral index becomes

$n_{s}=1+\frac{2\left\{\mathcal{H} \mathrm{cn} \Phi\left(1-2 \mathrm{dn}^{2} \Phi\right)+\left[\mathrm{cn}^{2} \Phi-3 \mathrm{dn}^{2} \Phi\left(\frac{5}{3} \mathrm{cn}^{2} \Phi-1\right)\right]\right\}}{[\mathcal{H}+\mathrm{cn} \Phi]^{2}}$. 


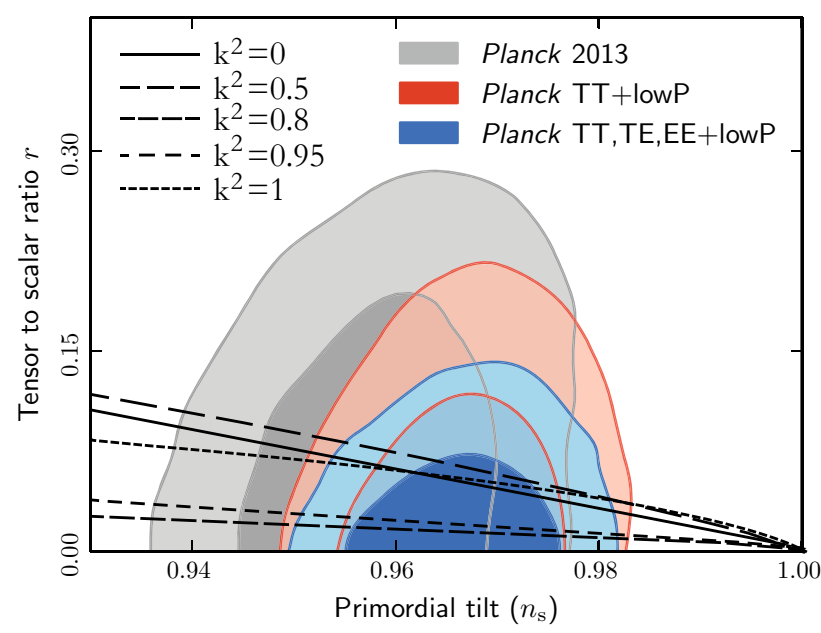

Fig. 5 This plot shows the parameter $r$ as a function of the scalar spectral index $n_{s}$ for various values of the modulus $k$, i.e. $k=0, k^{2}=$ $0.5, k^{2}=0.8, k^{2}=0.95$, and $k=1$. The marginalized joint 68 and $95 \%$ confidence level regions using Planck TT + lowP or Planck TT, TE, EE + lowP are shown [35]. Also, constraints from the Planck 2013 data release are shown for comparison. All curves have been plotted with $H_{1}=1, \mathcal{H}=1.3$, and they are normalized in such way that $r=0$ when $n_{s}=1$

It is well known that not only scalar curvature perturbations are generated during inflation. In addition quantum fluctuations generate transverse-traceless tensor perturbations [30], which do not couple to matter. Therefore they are only determined by the dynamics of the background metric. The two independent polarizations evolve like minimally coupled massless fields with spectrum

$\mathcal{P}_{\mathcal{T}}=\left.\frac{16 \pi}{m_{p}^{2}}\left(\frac{H}{2 \pi}\right)^{2}\right|_{a H=\widehat{k}}$.

In the same way as the scalar perturbations, it is possible to introduce the gravitational wave spectral index $n_{T}$ defined by $n_{T} \equiv \frac{\mathrm{d} \ln \mathcal{P}_{\mathcal{T}}}{\mathrm{d} \ln \hat{k}}$, which in our case becomes $n_{T}=-2 \epsilon_{H}$. At this point, we can introduce the tensor-to-scalar amplitude ratio $r \equiv \frac{\mathcal{P}_{\mathcal{I}}}{\mathcal{P}_{\mathcal{R}}}$, which becomes

$r=4 \epsilon_{H}$,

and, in our case, we obtain

$r=\frac{4 \mathrm{sn}^{2} \Phi \mathrm{dn}^{2} \Phi}{[\mathcal{H}+\mathrm{cn} \Phi]^{2}}$

In Fig. 5 we display the parameter space of $r$, given by Eq. (50), and the spectral index given by Eq. (47).

\section{Final remarks}

There is no doubt that elliptic functions are a powerful tool to describe natural phenomena. In this paper we have used them to describe a scalar field in order to find inflationary solutions. In particular, we have used an inflationary universe model in which the inflaton field is characterized by a parameter of the equation of state $\omega_{\phi}=-1+\sqrt{f_{\phi}}$, where $f_{\phi}$ is given by Eq. (23), and its kinematical evolution was described by the Hubble parameter given by $H(\phi)=H_{1}\left\{\mathcal{H}+\operatorname{cn}\left[\beta\left(\phi-\phi_{0}\right)\right]\right\}$. A detailed analysis shows that the allowed values for $H(\phi)$ depend strongly on the values of the pair $(k, \mathcal{H})$. On this basis, the scalar potential, $V(\phi)$, the corresponding number of e-foldings, and the attractor feature of the model were described.

We should mention here that the first hierarchy parameter $\epsilon_{H}$ (and thereby, the deceleration parameter $q$ ) related to the inflaton field results in such a way that it is possible to reproduce a generalized Chaplygin gas by making $\mathcal{H} \rightarrow 0$. This analogy encouraged us to start a generalization of the GCG in terms of elliptic functions [36].

It was also shown that the potential works quite well when compared to the measurements recently released based on Planck data. This situation is the main motivation to study inflationary universe models with this kind of scalar potential.

Finally, in general terms, we have found that the tensor-toscalar ratio can adequately accommodate the currently available observational data for some values of the $(\mathcal{H}, k)$ parameters. In this context, we have shown that the model here presented is appropriate for describing inflationary universe models.

Acknowledgments J. R. Villanueva dedicates this paper to his master and friend, Sergio del Campo. E. Gallo is thankful for kind hospitality at Instituto de Física y Astronomía, Universidad de Valparaíso, while working on this paper. We would like to thank Amelia Bayo, Víctor Cárdenas, Ramón Herrera, Osvaldo Herrera, and Cuauhtemoc Campuzano for helpful conversations relating to this work. E. Gallo acknowledges financial support from CONICET and SeCyT-UNC. J. R. Villanueva is supported by Comisión Nacional de Investigación Científica y Tecnológica through FONDECYT Grant No. 11130695.

Open Access This article is distributed under the terms of the Creative Commons Attribution 4.0 International License (http://creativecomm ons.org/licenses/by/4.0/), which permits unrestricted use, distribution, and reproduction in any medium, provided you give appropriate credit to the original author(s) and the source, provide a link to the Creative Commons license, and indicate if changes were made.

Funded by SCOAP ${ }^{3}$.

\section{Appendix A: A brief review of Jacobian elliptic functions}

As a starting point, let us consider the elliptic integral

$$
\begin{aligned}
u(y, k) & \equiv u=\int_{0}^{y} \frac{\mathrm{d} t}{\sqrt{\left(1-t^{2}\right)\left(1-k^{2} t^{2}\right)}} \\
& =\int_{0}^{\varphi} \frac{\mathrm{d} \theta}{\sqrt{1-k^{2} \sin ^{2} \theta}}=F(\varphi, k),
\end{aligned}
$$

where $F(\varphi, k)$ is the normal elliptic integral of the first kind, and $k$ is the modulus. The problem of the inversion of this 
integral was studied and solved by Abel and Jacobi, and this leads to the inverse function defined by $y=\sin \varphi=\operatorname{sn}(u, k)$ with $\varphi=$ am $u$, and the functions are called Jacobi elliptic sine $u$ and amplitude $u$.

The function sn $u$ is an odd elliptic function of order two. It possesses a simple pole of residue $1 / k$ at every point congruent to $i K^{\prime}\left(\bmod 4 K, 2 i K^{\prime}\right)$ and a simple pole of residue $-1 / k$ at points congruent to $2 K+i K^{\prime}\left(\bmod 4 K, 2 i K^{\prime}\right)$, where $K \equiv K(k)=F(\pi / 2, k)$ is the complete elliptic integral of the first kind, $K^{\prime}=F\left(\pi / 2, k^{\prime}\right)$, and $k^{\prime}=\sqrt{1-k^{2}}$ is the complementary modulus.

Two other functions can then be defined by $\operatorname{cn}(u, k)=$ $\sqrt{1-y^{2}}=\cos \varphi, \operatorname{dn}(u, k)=\sqrt{1-k^{2} y^{2}}=\Delta \varphi=$ $\sqrt{1-k^{2} \sin \varphi}$. The set of functions $\{\operatorname{sn} u, \operatorname{cn} u, \operatorname{dn} u\}$ are called Jacobian elliptic functions, and they take the following special values:

$\operatorname{sn}(u \mid 0)=\sin u, \quad \operatorname{sn}(u \mid 1)=\tanh u$,

$\operatorname{cn}(u \mid 0)=\cos u, \quad \operatorname{cn}(u \mid 1)=\operatorname{sech} u$,

$\operatorname{dn}(u \mid 0)=1, \quad \operatorname{dn}(u \mid 1)=\operatorname{sech} u$.

Some fundamental relations between Jacobian elliptic functions are

$\operatorname{sn}^{2} u+\operatorname{cn}^{2} u=1$,

$k^{2} \operatorname{sn}^{2} u+\operatorname{dn}^{2} u=1$,

$\mathrm{dn}^{2} u-k^{2} \mathrm{cn}^{2} u=k^{\prime 2}$,

$k^{\prime 2} \operatorname{sn}^{2} u+\operatorname{cn}^{2} u=\mathrm{dn}^{2} u$.

\section{References}

1. V.H. Cárdenas, Inflation as a response to protect the holographic principle. Mod. Phys. Lett. A 24, 29, 2353 (2009)

2. A.R. Liddle, P. Parsons, J.D. Barrow, Formalizing the slow roll approximation in inflation. Phys. Rev. D 50, 7222 (1994)

3. D.S. Salopek, J.R. Bond, Nonlinear evolution of long-wavelength metric fluctuations in inflationary models. Phys. Rev. D 42, 3936 (1990)

4. A.G. Muslimov, On the scalar field dynamics in a spatially flat Friedman universe. Class. Quantum Gravity 7, 231 (1990)

5. W.H. Kinney, A Hamilton-Jacobi approach to nonslow roll inflation. Phys. Rev. D 56, 2002 (1997)

6. B.J. Carr, J.E. Lidsey, Primordial black holes and generalized constraints on chaotic inflation. Phys. Rev. D 48, 543 (1993)

7. F.E. Schunck, E.W. Mielke, A new method of generating exact inflationary solutions. Phys. Rev. D 50, 4794 (1994)

8. S.V. Chervon, V.M. Zhuravlev, Exact solutions in cosmological inflationary models. Russ. Phys. J. 39(8), 776 (1996)

9. H.C. Kim, Exact solutions in Einstein cosmology with a scalar field. Mod. Phys. Lett. A 28, 1350089 (2013)

10. R.M. Hawkins, J.E. Lidsey, Inflation on a single brane-exact solutions. Phys. Rev. D 63, 041301 (2001)

11. S. del Campo, Approach to exact inflation in modified Friedmann equation. J. Cosmol. Astropart. Phys. 12, 005 (2012)
12. S. del Campo, Single-field inflation à la generalized Chaplygin gas. J. Cosmol. Astropart. Phys. 11, 004 (2013)

13. A.A. Chaadaev, S.V. Chervon, New class of cosmological solutions for a self-interacting scalar field. Russ. Phys. J. 56(7), 725 (2013)

14. T. Harko, F.S.N. Lobo, M.K. Mak, Arbitrary scalar-field and quintessence cosmological models. Eur. Phys. J. C 74, 2784 (2014)

15. Z.K. Guo, Y.S. Piao, R.G. Cai, Y.Z. Zhang, Inflationary attractor from tachyonic matter. Phys. Rev. D 68, 043508 (2003)

16. Z.K. Guo, H.S. Zhang, Y.Z. Zhang, Inflationary attractor in brane world scenario. Phys. Rev. D 69, 063502 (2004)

17. P.F. Byrd, M.D. Friedman, Handbook of Elliptic Integrals for Engineers and Scientists, 2nd edn. (Springer, Berlin, 1971)

18. J.V. Armitage, W.F. Eberlein, Elliptic Functions, London Mathematical Society Student Texts, vol. 67 (Cambridge University Press, Cambridge, 2006)

19. K. Meyer, Jacobi elliptic functions from a dynamical systems point of view. Am. Math. Mon. 108, 729 (2001)

20. H. Hancock, Theory of Elliptic Functions (Dover publications, New York, 1958)

21. I.S. Gradshteyn, I.M. Ryzhik, Table of Integrals, Series, and Products (Academic Press, New York, 2007)

22. V.N. Lukash, Production of phonons in an isotropic universe. Sov. Phys. JETP 52, 807 (1980). [Zh. Eksp. Teor. Fiz. 79, 1601 (1980)]

23. S.W. Hawking, The development of irregularities in a single bubble inflationary universe. Phys. Lett. B 115, 295 (1982)

24. A.A. Starobinsky, Dynamics of phase transition in the new inflationary universe scenario and generation of perturbations. Phys. Lett. B 117, 175 (1982)

25. L.P. Grishchuk, Amplification of gravitational waves in an istropic universe. Sov. Phys. JETP 40, 409 (1975). [Zh. Eksp. Teor. Fiz. 67, 825 (1974)]

26. A.A. Starobinsky, Spectrum of relict gravitational radiation and the early state of the universe. JETP Lett. 30, 682 (1979). [Pisma Zh. Eksp. Teor. Fiz. 30, 719 (1979)]

27. V.A. Rubakov, M.V. Sazhin, A.V. Veryaskin, Graviton creation in the inflationary universe and the grand unification scale. Phys. Lett. B 115, 189 (1982)

28. R. Fabbri, M.D. Pollock, The Effect of Primordially Produced Gravitons upon the Anisotropy of the Cosmological Microwave Background Radiation. Phys. Lett. B 125, 445 (1983)

29. L.F. Abbott, M.B. Wise, Constraints on generalized inflationary cosmologies. Nucl. Phys. B 244, 541 (1984)

30. V. Mukhanov, H. Feldman, R. Brandenberger, Theory of cosmological perturbations. Part 1. Classical perturbations. Part 2. Quantum theory of perturbations. Part 3. Extensions. Phys. Rep. 215, 203 (1992)

31. M. Kamionkowski, A. Kosowsky, A. Stebbins, A probe of primordial gravity waves and vorticity. Phys. Rev. Lett. 78, 2058 (1997)

32. L. Knox, Y. Song, A limit on the detectability of the energy scale of inflation. Phys. Rev. Lett. 89, 011303 (2002)

33. A.H. Guth, S.Y. Pi, Fluctuations in the new inflationary universe. Phys. Rev. Lett. 49, 1110 (1982)

34. J. Bardeen, P. Steinhardt, M. Turner, Spontaneous creation of almost scale-free density perturbations in an inflationary universe. Phys. Rev. D 28, 679 (1983)

35. P.A.R. Ade et al., Planck 2015 results. XX. Constraints on inflation. arXiv: 1502.02114

36. J.R. Villanueva, The generalized Chaplygin-Jacobi gas (2015). arXiv:1505.03107 